\title{
LEVANTAMENTO RÁPIDO DE PEIXES EM UMA LAGOA MARGINAL DO RIO IMBITUVA NA BACIA DO ALTO RIO TIBAGI, PARANÁ, BRASIL
}

\author{
A QUICK SURVEY OF THE FISH COMMUNITY IN A MARGINAL \\ LAGOON OF THE IMBITUVA RIVER, IN THE UPPER TIBAGI BASIN, \\ IN PARANÁ, BRAZIL
}

\author{
João Felipe Moutinho Sant'Anna', Mara Cristina de Almeida', \\ Marcelo Ricardo Vicari ${ }^{2}$, Oscar Akio Shibatta ${ }^{3}$, Roberto Ferreira Artoni ${ }^{*}$ \\ 1* Autor para contato: Universidade Estadual de Ponta Grossa - UEPG, Departamento de \\ Biologia Estrutural, Molecular e Genética, Campus em Uvaranas, Ponta Grossa, PR, \\ Brasil; e-mail: rfartoni@uepg.br \\ 2 Universidade Federal de São Carlos - UFSCAR, Programa de Pós-Graduação em \\ Genética e Evolução, São Carlos, SP \\ 3 Universidade Estadual de Londrina - UEL, Museu de Zoologia, Departamento de \\ Biologia Animal e Vegetal, PR
}

Recebido para a publicação em 31/10/05

Aceito para a publicação em 17/02/2006

\section{RESUMO}

O estudo da comunidade de peixes juvenis em uma lagoa marginal do Rio Imbituva foi realizado em um trecho próximo à foz (2507’13”'S e 50²3’20”'W), no período de abril a maio de 2005, por meio de duas coletas realizadas com peneirões utilizados na vegetação aquática e rede de arrasto em áreas limpas. Foram capturados 533 exemplares de peixes distribuídos em 7 espécies, pertencentes a 6 gêneros, 4 famílias e 2 ordens. Characiformes foi a ordem mais abundante, com maior número de representantes pertencentes à família Characidae. A espécie mais freqüente foi o lambari-prata Hyphessobrycon reticulatus, com 404 indivíduos. A espécie Mimagoniates microlepis foi encontrada pela primeira vez na bacia do rio Tibagi.

Palavras-chave: diversidade, conservação, characiformes, perciformes

\begin{abstract}
The juvenile fish community of a marginal lagoon of the Imbituva River was studied in a region close to its estuary ( $25^{\circ} 07^{\prime} 13^{\prime \prime}$ S e $\left.50^{\circ} 23^{\prime} 20^{\prime \prime} \mathrm{W}\right)$, through samples collected in April and May 2005. 533 individuals of 7 species, belonging


to 6 genera of 4 families and 2 orders, were collected. Characiformes was the most abundant order, with the largest number of representatives in the Characidae family. The most abundant among the species collected was Hyphessobrycon reticulatus, with 404 individuals. The species Mimagoniates microlepis was captured for the first time in the Tibagi River basin.

Key words: diversity, conservation, characiformes, perciformes

\section{Introdução}

Lagoas marginais de sistemas de rio-planície de inundação são amplamente reconhecidas pela sua importância na manutenção e integridade da biodiversidade regional, seja como criadouros naturais das espécies de importância comercial, na sua maioria migradores de longa distância (Agostinho et al., 1993; 2000), ou como hábitat preferencial das espécies sedentárias e de pequeno porte. A diversidade ictiofaunística e o tamanho dos estoques das espécies migradoras dependem, em grande parte, da conservação e integridade das lagoas marginais (Petry et al., 2002).

Nos sistemas tropicais, a reprodução de algumas espécies de peixes que vivem em rios com planície de inundação é altamente sazonal e, para a maioria delas, coincide com as primeiras fases de cheia (Welcomme, 1979; Araújo-Lima et al., 1995). As enchentes funcionam como gatilho sincronizador para desova, e o pico da enchente, como finalizador do período reprodutivo (Vazzoler et al., 1997). Durante as cheias, os rios restabelecem a sua conexão com as lagoas marginais, fornecendo condições necessárias para o incremento da sua produtividade biológica (Junk et al., 1989). Com a reprodução ocorrendo nesse momento anual, os peixes aumentam a probabilidade dos novos indivíduos terem acesso aos hábitats que serão os seus berçários, lhes oferecendo proteção e alimento (Lowe McConnell, 1975; Welcomme,1979).

Alguns relatos especulam acerca do grande berçário que o rio Imbituva representa à bacia do rio Tibagi, em função das inúmeras lagoas marginais existentes ao longo de seu curso. Assim, o presente trabalho tem o objetivo de investigar de modo rápido a ocorrência de peixes em lagoa marginal ao rio Imbituva, um dos principais afluentes do rio Tibagi.

\section{Material e Métodos}

Do contato etnológico com pescadores regionais constatou-se que o rio Imbituva e suas lagoas marginais poderiam representar um local de importância para a conservação de peixes na bacia do rio Tibagi. Deste modo elaborou-se como desenho experimental o levantamento de peixes em uma lagoa marginal ao rio Imbituva por meio de duas coletas nos meses de abril e maio de 2005, anterior ao período de cheia e re-conexão com o rio Tibagi, com vistas a evidenciar a ocorrência de juvenis de peixes possibilitando testar o potencial deste tipo de ambiente para as primeiras fases do ciclo de vida de peixes residentes e migratórios. A lagoa escolhida para a prática possui forma de ferradura, o que possibilita duas conexões com o rio, no período de enchentes.

Cada coleta teve duração de cinco horas no período diurno, alternando os métodos de coleta. Como o objetivo das coletas foi o de proporcionar um levantamento das espécies em fase juvenil na lagoa, peneirões e rede de arrasto foram utilizadas como instrumentos. Como a lagoa possui vegetação aquática principalmente nas margens, o peneirão é indicado para a amostragem, o qual consiste numa rede de malha $5 \mathrm{~mm}$ presa a um aro. Já nas áreas rasas e desprovidas de vegetação utilizou-se uma rede de arrasto, eficiente na captura de juvenis, com malha fina ( 0,3 a 0,5 cm entre nós opostos) e comprimento de $5 \mathrm{~m}$.

Depois de coletados, os exemplares foram imediatamente preservados em formol a $10 \%$ para que a coloração original fosse mantida com o objetivo de obter registro fotográfico de exemplares testemunhos. Em laboratório, os lotes foram preservados em álcool a 70\%. Os exemplares foram depositados no Museu de Zoologia da Universidade Estadual de Londrina. 
A diversidade de espécies foi medida através do índice de diversidade de Shannon-Wiener $\left(\mathrm{H}^{\prime}=\mathrm{Spi} \mathrm{x}\right.$ lnpi, onde p é a freqüência relativa da espécie i), e o teste $t$ foi aplicado para verificar se havia diferença significativa ente os índices de diversidade. Tais análises foram conduzidas com o auxílio do programa PAST 1.34, segundo Hammer (2001).

\section{Área de estudo}

A área utilizada para o estudo foi uma lagoa entre diversas lagoas marginais existentes no rio Imbituva (2507'13"S 50²3’20"W) (Figura 1). O rio Imbituva apresenta uma grande extensão de área alagada ou alagável no período de chuva, devido a seu perfil "serpenteado” por inúmeras curvas. Tal modelo possibilita a formação de lagoas marginais por meandros abandonados em momentos de mudança do curso do rio.

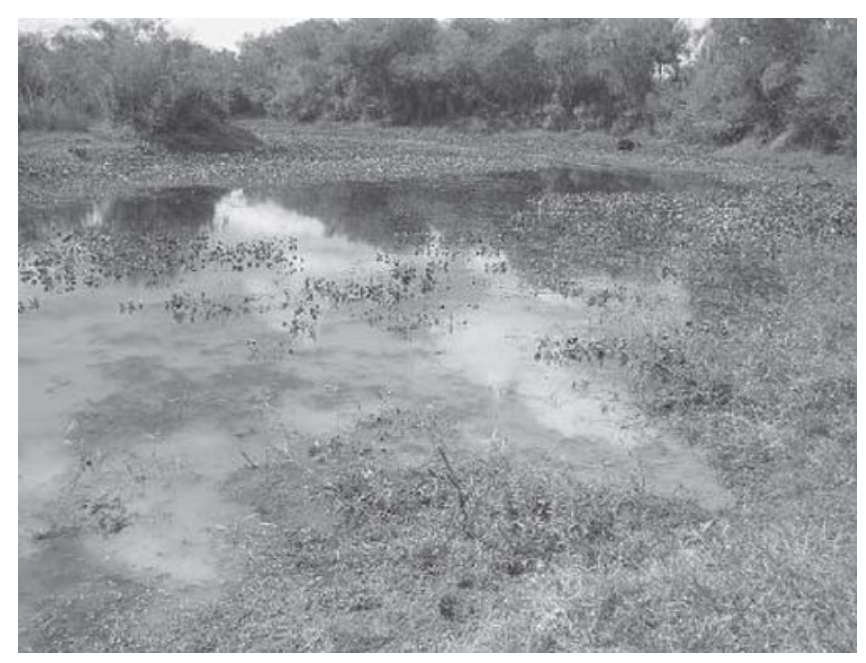

Figura 1 - Lagoa marginal ao rio Imbituva (Local de coleta).

Orio Imbituva éum dos 65 afluentes do rio Tibagi (Figura 2). Na área amostrada da bacia, localizada no Segundo Planalto paranaense, tanto o rio Tibagi como seus afluentes são influenciados fortemente pela ação antrópica da agricultura e pecuária que invadem e degradam a mata ciliar chegando, muitas vezes, até a margem do rio, provocando assoreamento e lixiviação de agrotóxicos. A vegetação que se apresenta no trecho do Alto Tibagi é o de Cerrado gramíneo-lenhoso e de tensão ecológica entre Cerrado e Floresta de Araucária.
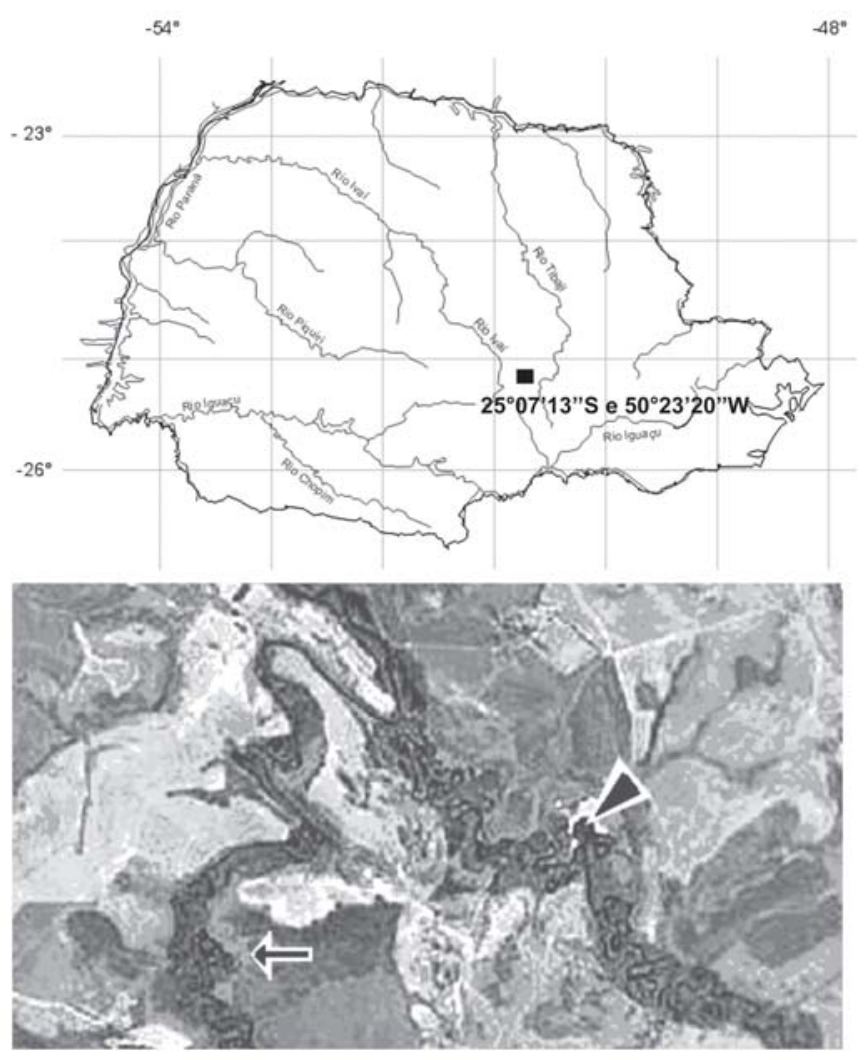

Figura 2 - Mapa hidrográfico do Paraná destacando sítio de coleta no rio Imbituva (seta) na bacia do alto rio Tibagi (cabeça de seta). Em detalhe imagem de satélite com localização do trecho estudado.

\section{Resultados e Discussão}

Foram coletados 533 exemplares de peixes, pertencentes a duas ordens (Characiformes e Perciformes), 4 famílias, 6 gêneros e 7 espécies. A relação das espécies tratadas neste trabalho é apresentada através do enquadramento taxonômico segundo Lauder e Liem (1983). Das espécies coletadas, aproximadamente 93,8\% pertencem à família Characidae, 4,7\% a Erythrinidae, 1,3\% a Crenuchidae e 0,2\% a Cichlidae (Tabela 1 e Figura 3). A dominância de Characiformes, principalmente família Characidae, é comum aos rios neotropicais.

Lista das espécies coletadas em uma lagoa marginal ao rio Imbituva, Paraná Brasil, segundo a classificação taxonômica de Lauder e Liem (1983): 


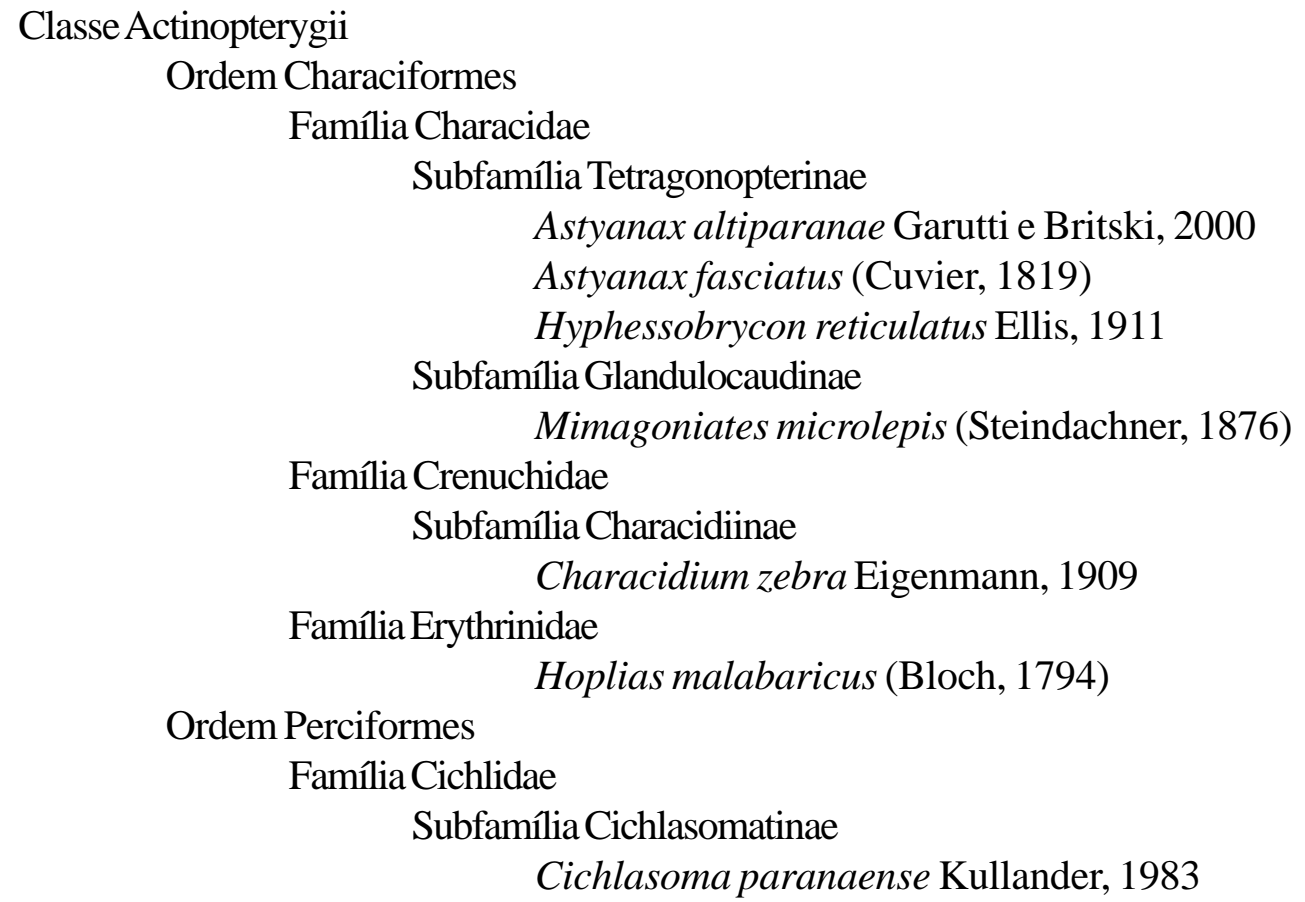

\begin{tabular}{ccccc}
\hline & \multicolumn{2}{c}{ Coleta 1 } & \multicolumn{2}{c}{ Coleta 2 } \\
\hline Espécies & Freq. abs. & Freq. rel & Freq. abs. & Freq. rel \\
\hline Hoplias malabaricus & 17 & 0,09 & 8 & 0,02 \\
Hyphessobrycon reticulatus & 148 & 0,77 & 256 & 0,75 \\
Astyanax altiparanae & 3 & 0,02 & 1 & 0,00 \\
Astyanax fasciatus & 18 & 0,09 & 66 & 0,19 \\
Mimagoniates microlepis & 6 & 0,03 & 2 & 0,01 \\
Characidium zebra & 1 & 0,01 & 6 & 0,02 \\
Cichlasoma paranaense & 0 & 0,00 & 1 & 0,00 \\
\hline Total & 193 & 1 & 340 & 1 \\
\hline
\end{tabular}

Tabela 1 - Freqüência das espécies coletadas na lagoa marginal ao rio Imbituva.

Com relação ao tamanho dos exemplares coletados, Hoplias malabaricus foi a espécie de maior comprimento padrão médio $(67,5 \mathrm{~mm})$ e Hyphessobrycon reticulatus (24 mm) a de menor comprimento. Todos os exemplares amostrados representam formas juvenis das espécies ocorrentes (Figura 3, Tabela 2).

Entre as duas coletas realizadas foi obtida uma dominância em número de exemplares coletados para Hyphessobrycon reticulatus, com 404 exemplares. Outra espécie que obteve presença considerável nas assembléias foi Astyanax fasciatus com 84 exem- plares. Comparando as duas coletas, calculou-se o índice de diversidade específica de Shannon-Wiener $\mathrm{H}^{\prime}=0,84$ e 0,75 , respectivamente para as coletas $1 \mathrm{e}$ 2. Esses valores são significativamente diferentes, conforme $o$ teste $t(t=0,82476, \mathrm{p}=0,41004)$.

Destaca-se também entre as duas coletas realizadas a diminuição do número de exemplares amostrados da coleta 1 para a coleta 2 para as espécies Hoplias malabaricus, Astyanax altiparanae e Mimagoniates microlepis. Sendo que na coleta 2, evidencia-se a presença de uma sétima espécie, Cichlasoma paranaense. 

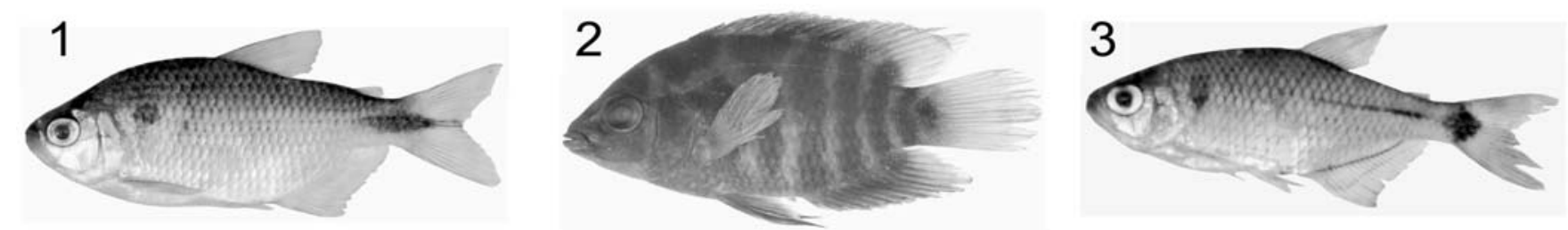

4

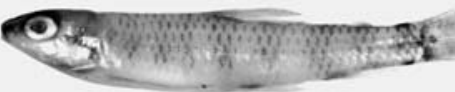

5

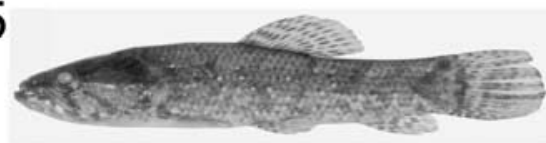

6
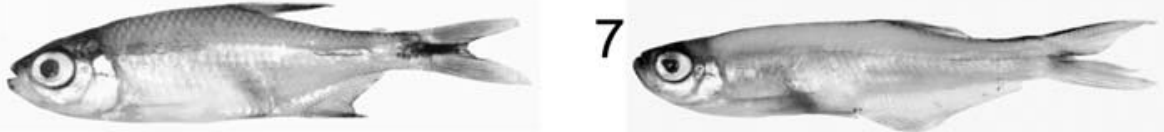

Figura 3 - Exemplares representativos das espécies das ordens Characiformes e Perciformes, coletadas na lagoa marginal do rio Imbituva. Os nomes científicos e populares das espécies são: 1) Astyanax altiparanae, lambari-relógio; 2) Cichlasoma paranaense, acará vovó; 3) Hyphessobrycon reticulatus, lambari-prata; 4) Characidium zebra, piquira; 5) Hoplias malabaricus, traíra; 6) Astyanax fasciatus, lambari do rabo vermelho; 7) Mimagoniates microlepis, tetra azul.

\begin{tabular}{lccc}
\hline \multicolumn{1}{c}{ Espécie } & N amostral & $\begin{array}{c}\text { Comprimento } \\
\text { Padrão }(\mathbf{c m})\end{array}$ & $\begin{array}{c}\text { Desvio Padrão da } \\
\text { Média }(\mathbf{c m})\end{array}$ \\
\hline Hoplias malabaricus & 25 & 4,4 & $\pm 1,3$ \\
Hyphessobrycon reticulatus & 404 & 3,3 & $\pm 1,0$ \\
Astyanax altiparanae & 4 & 4,3 & $\pm 1,6$ \\
Astyanax fasciatus & 84 & 2,3 & $\pm 0,3$ \\
Mimagoniates microlepis & 8 & 2,6 & $\pm 0,3$ \\
Characidium zebra & 7 & 2,1 & $\pm 0,3$ \\
Cichlasoma paranaense & 1 & 3,2 & - \\
\hline
\end{tabular}

Tabela 2 - Variação do comprimento padrão das espécies coletadas na lagoa marginal ao rio Imbituva.

O momento exato e o local da desova adequado garantem o sucesso reprodutivo e, conseqüentemente, a manutenção do tamanho da poulação (Munro 1990). Na maioria dos rios, poucas espécies se reproduzem ao longo de todo ano. O pico da desova geralmente ocorre entre dezembro e janeiro, no início da temporada de cheias (Lamas, 1993; Alves et al., 1998; Luz et al., 2000).

As lagoas de várzeas de grandes rios são bem conhecidas pela sua importância como criadouros naturais para peixes de piracema (Welcomme, 1979; LoweMcConnell, 1987; Welcomme, 1995; Bayley e Li, 1996). Após a fertilização, as larvas desenvolvem as estruturas corporais que as auxiliam na sobrevivência, podendo explorar os micro-habitats das lagoas para alimentação e refúgio de predadores.
No momento em que as coletas foram realizadas, não havia mais conexão entre o rio e a lagoa, tornandose um ambiente único à lagoa. Desta maneira, este ambiente pode oferecer condições para que as populações presentes se desenvolvam. Foi verificada a dominância da espécie Hyphessobrycon reticulatus, a qual possui características mais amplas e generalistas como a dieta onívora, a resistência a baixos níveis de oxigênio na água e ainda possuir alto potencial reprodutivo, além de permanecer em meio à vegetação aquática, o que poderia ter possibilitado uma maior defesa a ataques de predadores. Estes fatores podem ter levado ao aumento da população e dominância em relação às outras espécies desta comunidade.

A maioria das espécies coletadas não possui caráter migratório permanecendo todo o ciclo na lagoa. 
Apenas Astyanax altiparanae, Astyanax fasciatus e Characidium zebra são espécies migradoras não obrigatórias a curtas distâncias.

Foi verificada alteração na altura do nível da água da coleta 1 para a coleta 2 que diminuiu devido à falta de conexão entre a lagoa e o rio, e a baixa pluviosidade no período do estudo. Porém, o número de exemplares coletados na coleta 2 foi maior do que na coleta 1 . Acredita-se que seja pelo adensamento das populações no ambiente, o que indica pontos negativos para algumas espécies pela maior disputa de recursos. A Figura 4 ilustra as posições de cada espécie de peixe na coluna d'água.

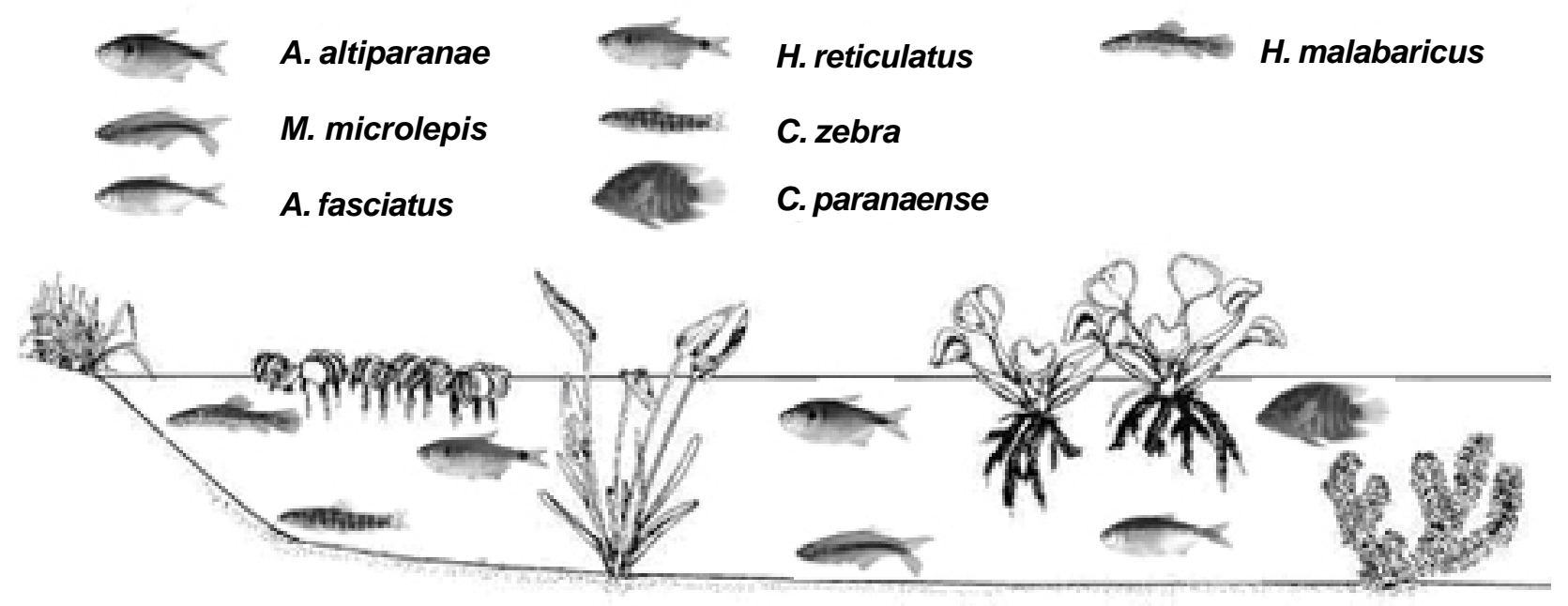

Figura 4 - Ilustração da distribuição dos peixes coletados na coluna d'agua na lagoa amostrada.

Os diferentes métodos empregados em coleta possibilitaram vislumbrar diferenças nos hábitos de cada espécie. Com o peneirão foram coletados exemplares de Hyphessobrycon reticulatus, Hoplias malabaricus e na segunda coleta, um exemplar de Cichlasoma paranaense foi amostrado. Já com a rede de arrasto foi possível coletar Astyanax altiparanae, Astyanax fasciatus, Mimagoniates microlepis, Hyphessobrycon reticulatus e Characidium zebra. A presença de $H$. reticulatus nas diferentes partes da coluna d'água evidencia sua dominância no ambiente, sugerindo que esta espécie consegue explorá-lo em maior dimensão.

As ordens Characiformes e Siluriformes segundo Lowe-McConnel (1987) e Bennemann et al. (2000), são mais freqüentes para os rios sul-americanos e no próprio rio Tibagi, respectivamente. Porém, sobre a ausência de exemplares da ordem Siluriformes nas coletas, acredita-se que esteja relacionada especialmente com os horários de coleta. Neste trabalho as coletas foram diurnas, o que se contrapõe aos hábitos das espécies desta ordem. Geralmente, estas espécies são capturadas em coletas noturnas com redes de espera, onde atingem o pico de atividade, pois se alimentam com maior freqüência durante a noite. Contudo, como a vegetação marginal foi explorada seria esperada a presença de siluriformes de pequeno porte comuns na região a exemplo de Corydoras paleatus, o que não ocorreu. Segundo Araújo-Lima et al. (1995) e Bennemann et al. (2000), outro fator reside em que Characiformes predominam em ambientes de baía e margens, enquanto que em ambientes próximos ao canal do rio e fundo encontram-se Siluriformes de grande porte, o que não caracterizou as coletas deste trabalho, as quais foram realizadas em lagoa marginal.

Grande parte dos indivíduos coletados são formas juvenis das diferentes espécies evidenciadas pelo comprimento padrão médio verificado entre os exemplares registrados (Tabela 2), destacando a importância deste ambiente para a permanência durante os primeiros estágios de vida de algumas espécies de peixes regionais.

É necessário considerar mais enfaticamente a ocorrência de Mimagoniates, como uma espécie possivelmente de distribuição mais ampla do que aquela verificada até o momento. Trata-se de uma espécie de lambari da subfamília Glandulocaudinae eque segundo 
Menezes e Weitzman(1990) é endêmica a rios e riachos da Floresta Atlântica. Shibatta (comunicação pessoal) enfatiza que em esforços de coletas anteriores já realizados no rio Imbituva, demonstraram ausência da espécie o que coloca esta situação como uma questão ainda em aberto em relação a real distribuição e biogeografia histórica desses peixes.

A reduzida ocorrência de espécies migradoras nas lagoas marginais do rio Imbituva, pelo menos em relação a espécies de não siluriformes, destaca a relevância de ampliar esta modalidade de estudo para avaliar a condição das lagoas marginais ao rio Imbituva nos processos de reprodução destas espécies. Por outro lado, evidencia a importância dos ambientes das lagoas marginais na manutenção e para o desenvolvimento de espécies locais e migradoras a curtas distâncias. Apesar do seu caráter preliminar o presente trabalho descreve a ocorrência de uma espécie (Mimagoniates microlepsis) ainda não reconhecida na bacia, demonstrando a relevância de estudos desta natureza para o melhor entendimento da ictiofauna regional.

Agradecimentos

Ao Instituto Ambiental do Paraná e Instituto Brasileiro do Meio Ambiente pela licença para captura de peixes e a Universidade Estadual de Ponta Grossa pelo apoio logístico. Somos particularmente gratos a Dra M. L. Leite pelo apoio na estatística e aos senhores M. A. Carvalho, E. R. Sepka e R. T. Zenardi pelo apoio técnico.

\section{REFERÊNCIAS}

01. AGOSTINHO, A.A., THOMAZ, S.M., MINTE-VERA, C.V. \& WINEMILLER, K.O. Biodiversity in the high Paraná River floodplain. In: GOPAL, B.; JUNK, W.J.; DAVIS, J.A. Biodiversity in wetlands: assessment, function and conservation, Backhuys Publishers: Leiden, The Netherlands. 2000. p.89-118.

02. AGOSTINHO, A. A., VAZZOLER, A.E.A. de M., GOMES, L.C. \& OKADA, E.K. Estratificación espacial y comportamiento de Prochilodus scrofa em distintas fases del ciclo de vida, em la planície de inundación del alto rio Paraná y embalse de Itaipu, Paraná, Brasil. ver. d’Hydrobiol. Trop., v.26, n 1, p.79-90, 1993.

03. ALVES, C.M.B., VIEIRA, F., POMPEU, P. \& FONSECA,
P.R. Estudos de ictiofauna. In: Plano Diretor de recursos hídricos das bacias afluentes do rio São Francisco em Minas Gerais, Belo Horizonte: Governo Federal de Minas Gerais. Vol.1, Tomo IV. 1998. 154p.

04. ARAÚJO-LIMA, C.A.R.M., AGOSTINHO, A.A. \& FABRÉ, N.N. Trophic aspects of fish communities in Brazilian rivers and reservois. In: TUNDISI, J.G.; BICUDO, C.E.M.; MATSUMURA-TUNDISI, T. Limnology in Brazil, ABC/SBL, Rio de Janeiro. 1995. p. 105-136.

05. BAYLEY P.B. \& LI, H.W. River biota: diversity and dynamics. Blackwell Science, London. 1996. 257p.

06. BENNEMANN, S.T., SHIBATTA, O.A. \& GARAVELLO, J.C. Peixes do rio Tibagi: uma abordagem ecológica. Ed. UEL, Londrina. 2000.62p.

07. HAMMER, Ø., HARPER, D.A.T. \& RYAN, P. D. PAST: Paleontological Statistics Software Package for Education and Data Analysis. Palaeontologia Electronica v.4, n 1, 9pp, 2001.

08. JUNK, W.J., BAYLEY, P.B. \&APARKS, R.E. The flood pulse concept in river-floodplain systems. Can. J. Fish. Aquat. Sci., v.106, p.110-127, 1989.

09. LAMAS I.R. Análise de características reprodutivas de peixes brasileiros de água doce, com ênfase no local de desova. Dissertação, Mestrado em Ecologia, Conservação e Manejo de Vida Silvestre: Instituto de Ciências Biológicas, Universidade Federal de Minas Gerais. Belo Horizonte, 1993. 72p.

10. LAUDER, G.V. \& LIEM, K.F. The evolution and interrelationships of the Actinopterygian fishes. Bull. Mus. Comp.Zool. 150(3):95-197.1983.

11. LOWE-McCONNELL, R.H. Fish communities in tropical freshwaters: their distribution, ecology and evolution. Longman, London. 1975. 337p.

12. LOWE-McCONNELL, R.H. Ecological studies in tropical fish communities. Cambridge University Press, London. 1987. 382p.

13. LUZ, J.S., GODINHO, A. \& PETRERE Jr, M. As desovas de peixes no Alto-Médio São Francisco. Minas Gerais, Brasil. Universidade Federal de Minas Gerais. 2000.

14. MENEZES, N.A. \& MENEZES, S.H. Two new species of Mimagoniates (Teleostei, Characidae, Glandulocaudinae), their phylogeny and biogeography and a key to glandulocaudin fishes of Brazil and Paraguay. Proceedings of Biological Society of Washington. 1990, p.380-426.

15. MUNRO, A.D. General introduction. P.1-11. In: MUNRO A.D., SCOTT A.P. \& T.J. LAM Reproductive seasonality in teleosts environmental influences. crc Press, Florida. 1990. 254p.

16. PETRY, A.C., ABUJANRA, F., PIANA, P.A., JÚLIO JR, H.F. \& AGOSTINHO, A.A. Assembléias de Peixes das Lagoas 
Sazonalmente isoladas da Planície de Inundação do Alto Rio

Paraná. Maringá, PR. Universidade Estadual de Maringá, Nupélia/PEA. 2002.

17. VAZZOLER, A.E.A.M, AGOSTINHO, A.A. \& HAHN, N.S. A Planície de Inundação do Alto Rio Paraná. : EDUEM,
Maringá. 1997, p.267-280.

18. WELCOMME, R.L. Fisheries ecology of floodplain rivers. Longman, New York. 1979. 317p.

19. WELCOMME, R.L. Relationships between fisheries and the integrity of river systems. Reg. Riv. Res. Man. 11: 121-136. 1995. 